\title{
Automatic Fault Diagnosis Method for Wind Turbine Generator Systems Driven by Vibration Signals
}

\author{
Yu Pang ${ }^{\mathrm{a}, *}$, Limin Jia ${ }^{\mathrm{a}}$, Zhan $\mathrm{Liu}^{\mathrm{a}}$, and Qianyun Gao ${ }^{\mathrm{b}}$ \\ ${ }^{a}$ State Key Lab of Rail Traffic Control and Safety, Beijing Jiaotong University, Beijing, 100044, China \\ ${ }^{b}$ Beijing Nego Automation Technology Co. Ltd., Beijing, 10044, China
}

\begin{abstract}
An automatic fault diagnosis method for the wind turbine generator system (WTGS) driven by vibration signal is proposed in this paper. In this method, the vibration signal is used to drive the notch filter network directly, and the frequency selection characteristics of the notch filter are used to extract the fault feature frequency of WTGS components. Then, the extracted fault feature frequency is encoded and a neural network classifier is used to achieve the automatic fault diagnosis of WTGS. In addition, the vibration intensity is calculated to evaluate the fault degree of the WTGS. The innovation of this paper is that the fault feature frequency of the WTGS is derived from parameters of the notch filter rather than the vibration signal itself. The practical on-site application shows the effectiveness of the proposed method, which is of great significance for improving the efficiency of fault diagnosis of WTGS and realizing the batch diagnosis of the fault of WTGS.
\end{abstract}

Keywords: automatic fault diagnosis; notch filter; wind turbine generator system; vibration intensity; neural network

(Submitted on March 8, 2018; Revised on April 12, 2018; Accepted on May 2, 2018)

(C) 2018 Totem Publisher, Inc. All rights reserved.

\section{Introduction}

In recent years, wind power generation has been developing rapidly and has achieved large-scale commercial application. According to national industrial planning, the wind power industry will still have great developmental space in the future. Wind power generation is a typical distributed power generation system, which has the characteristics of small stand-alone capacity, wide distribution area, and the capability of servicing severe environments [7,6]. How to efficiently and reliably diagnose the fault in a wind turbine generator system (WTGS) and reduce downtime of the system has great practical significance in improving the operation and maintenance efficiency and economic benefits of wind farms.

Presently, the fault diagnosis of WTGS mainly depends on the manual operation. Through analyzing and processing the vibration signals of key components of WTGS, the fault feature frequency of the key components of the system is obtained, and the fault diagnosis is performed accordingly. The disadvantage of this fault diagnosis method is inefficiency, which cannot effectively support the large-scale fault diagnosis of WTGS, and thus, cannot efficiently improve the operation and maintain the efficiency of the wind farm $[4,3,9]$.

For the problem of low efficiency of fault diagnosis for the WTGS, some scholars have proposed automatic fault diagnosis technology. For most of these automatic fault diagnosis technologies, the fault feature information of the components is obtained first based on the analysis and processing of vibration signals, and then the automatic fault diagnosis is carried out according to the training of neural networks through fault feature information. Although this kind of automatic fault diagnosis technology can realize automation of fault diagnosis to a certain extent, it needs a large amount of calculations and a great deal of historical data to support it. These are basically off-line modes and cannot quickly support the fault diagnosis of WTGS.

\footnotetext{
* Corresponding author.

E-mail address: yupang_nego@126.com
} 
Aimed at the inefficiency of the fault diagnosis of WTGS, an automatic fault diagnosis method for WTGS driven by vibration signal and an automatic fault diagnosis method for WTGS based on the notch filter network are constructed. The innovation of this method is that the fault feature frequency of the WTGS component is not extracted from the vibration signal itself, but from the notch frequency parameter of the notch filter.

\section{Method}

\subsection{System Architecture}

The system structure of the automatic fault diagnosis method for WTGS based on notch filter is shown in Figure 1 below:

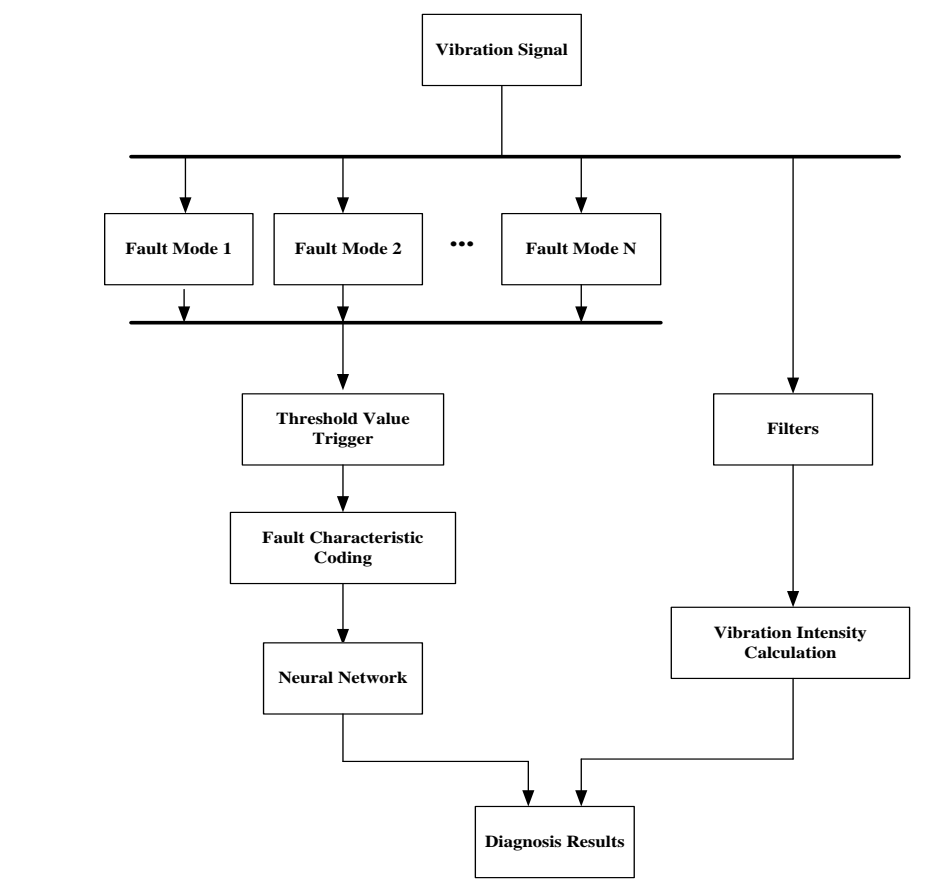

Figure 1. Structure of automatic fault diagnosis method for WTGS based on notch filter

As shown in Figure 1, the automatic fault diagnosis method for WTGS based on notch filter can automatically identify the typical faults of core components of the WTGS. At the same time, the fault degree of the core components of the system can be evaluated based on the vibration intensity index. The typical faults of the core components of WTGS can be reflected by a combination of one or several feature frequencies. As long as one or more specific frequency components can be automatically identified from the time-domain vibration signal, the automatic fault diagnosis can be realized. In this paper, a frequency-selection network based on notch filter is designed to realize automatic identification of the fault feature frequency. The special feature of this method is that the fault feature frequency is taken from the cut-off frequency of the notch filter rather than the vibration signal itself. Through coding of different combinations of different fault feature frequencies of the WTGS, the automatic classification of the neural network is used to achieve automatic fault diagnosis of the WTGS. In addition, according to the WTGS vibration standard VDI3834, the vibration intensity of the WTGS vibration signal is calculated to realize evaluation of the fault degree of WTGS based on the vibration intensity parameter.

\subsection{Working Principle of Frequency-selection Network based on Notch Filter}

Notch filter can filter the signal of a specific frequency component without affecting the signal of other frequency components [2]. Its transfer function is as follows:

$$
\frac{s^{2}+2 \xi_{1} \omega_{1}+\omega_{1}^{2}}{s^{2}+2 \xi_{2} \omega_{2}+\omega_{2}^{2}}
$$

If $\xi_{1}=0$ and $\omega_{1}=\omega_{2}$ in the above formula, the following formula can be established: 


$$
\frac{s^{2}+\omega^{2}}{s^{2}+2 \xi \omega+\omega^{2}}
$$

The frequency characteristics are shown in Figure 2 below, and as shown in Figure 2, the notch filter eliminates the signal of a particular frequency (notch frequency) in the original signal without affecting the signal of other frequencies. If the original signal subtracts the signal that has passed through the notch filter, a notch frequency signal will be obtained. Based on this principle, a frequency-selection network based on notch filter is designed, as shown in Figure 3. After the vibration signal passes through the frequency-selection network, a series of notch signals will be obtained, and the frequency of these notch signals is the same as the notch frequency of the notch filter. If the fault feature frequency of WTGS is set as the notch frequency of notch filter, when the vibration signal is input to the frequency-selection network shown in Fig. 3 , the identification of fault feature frequency of WTGS can be realized through extraction of the notch frequency of the notch filter as long as it can determine which notch filter is triggered. Therefore, it solves the problem of extracting fault feature frequency quickly and efficiently in automatic fault diagnosis.

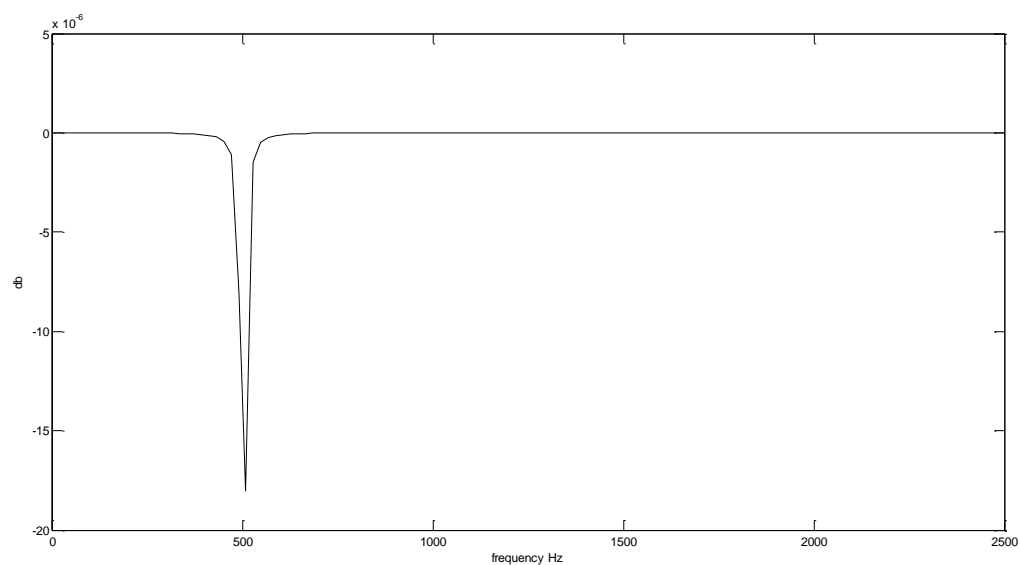

Figure 2. Frequency-domain characteristics of notch filter

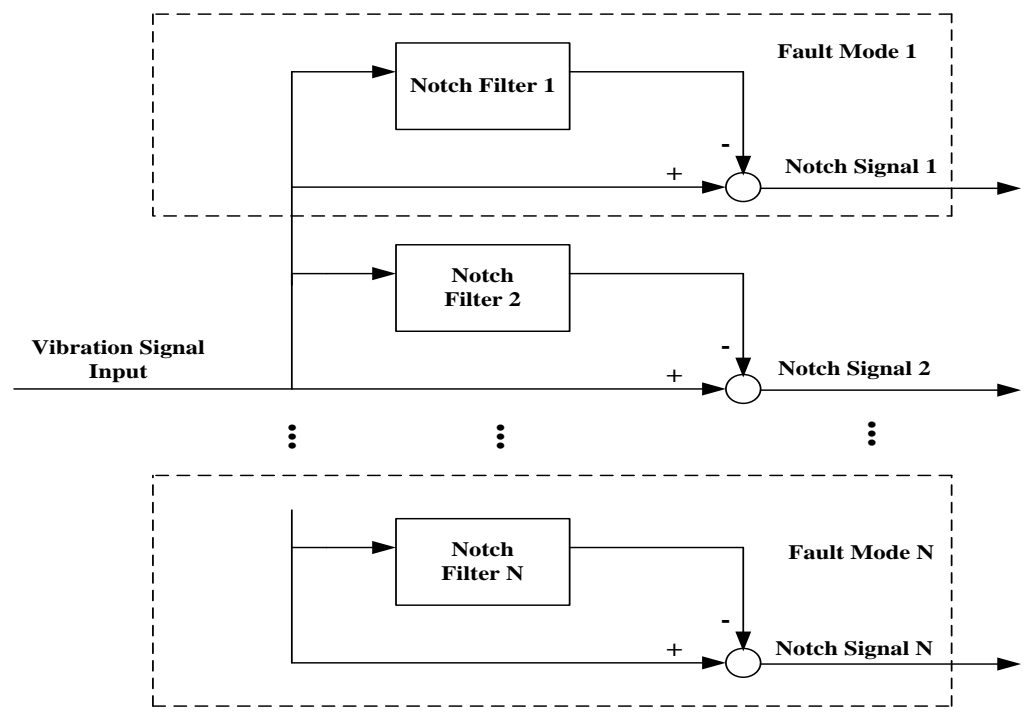

Figure 3. Frequency-selection network based on notch filter

\subsection{Triggering of Notch Filter and Fault Feature Encoder}

The energy of the notch signal and the percentage of the energy of the notch signal to that of the original vibration signal are calculated respectively. The order is arranged according to the ratio of the energy of different notch signals to that of the original vibration signal. When the energy percentage of the notch signal is greater than the set threshold, it is considered that 
the notch filter is triggered, and the notch frequency of the triggered notch filter is identified as the fault feature frequency. Based on the types of WTGS components and their typical fault characteristics, the fault feature frequency identified by the frequency-selection network is encoded. The process is shown in Figure 4 below.

The energy calculation formula of the notch signal is as follows [1]:

$$
A_{i}=\sum_{k=1}^{K} x_{i}^{2}(k)
$$

Where $A_{i}$ represents the energy of the $i-t h$ notch signal, and $x_{i}(k)$ represents the value of the $i-t h$ notch signal at the time of $k$.

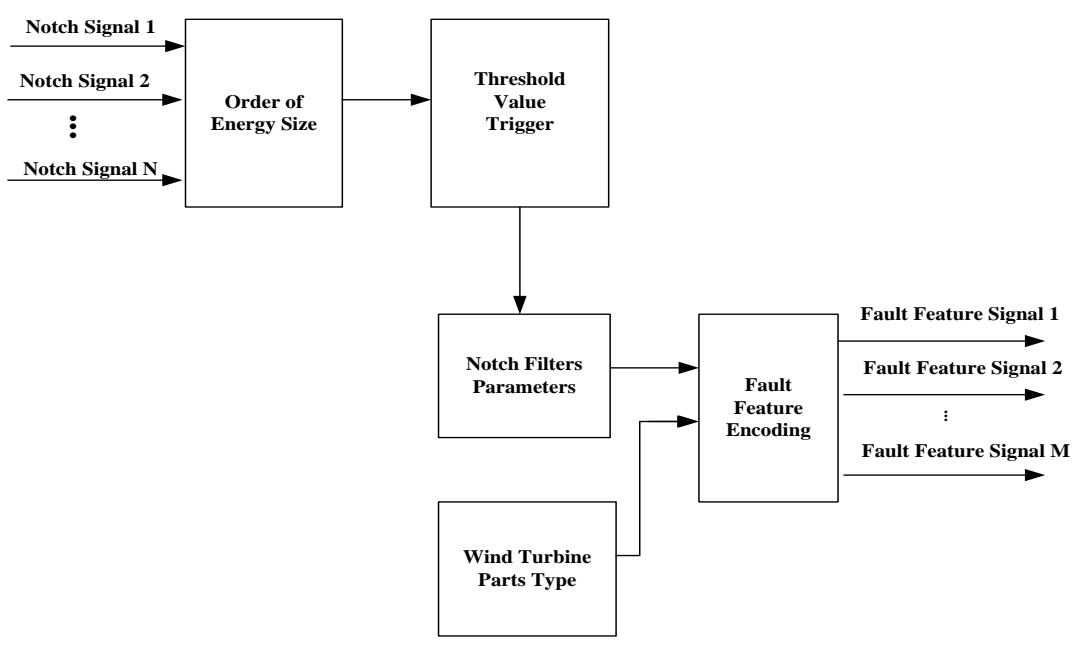

Figure 4. Schematic diagram of notch filter triggering and fault feature coding

Different feature frequencies are focused for different types of WTGS components. Therefore, selecting different feature frequencies for different components will help simplify the frequency-selection network and improve the efficiency of diagnosis, as shown in Table 1 below:

Table 1. Sample table of fault features

\begin{tabular}{|c|c|c|c|c|c|}
\hline Feature frequency & Generator & Gearbox & Shaft coupling & Nacelle /Tower & Remarks \\
\hline $1 \mathrm{P}$ & l & l & / & $\sqrt{ }$ & Rotation frequency of low-speed shaft \\
\hline $2 \mathrm{P}$ & 1 & 1 & 1 & $\sqrt{ }$ & 1 \\
\hline $3 \mathrm{P}$ & l & I & l & $\sqrt{ }$ & l \\
\hline $1 \mathrm{X}$ & $\sqrt{ }$ & $\sqrt{ }$ & $\sqrt{ }$ & l & Rotation frequency of high-speed shaft \\
\hline $2 X$ & $\sqrt{ }$ & 1 & $\sqrt{ }$ & I & 1 \\
\hline $5 X$ & $\sqrt{ }$ & 1 & 1 & I & I \\
\hline GMF & / & $\sqrt{ }$ & / & l & Gear mesh frequency \\
\hline $2 \mathrm{GMF}$ & I & $\sqrt{ }$ & 1 & 1 & 1 \\
\hline 3GMF & I & $\sqrt{ }$ & 1 & 1 & 1 \\
\hline LF & $\sqrt{ }$ & 1 & 1 & 1 & Power grid frequency \\
\hline
\end{tabular}

The generator fault shown in Table 1 is related to the rotation frequency of the generator system and power grid frequency. To diagnose faults in a generator, it only needs to pay attention to the rotation frequency of the generator and frequency of the grid, and there is no need to care about feature parameters such as the mesh frequency of gearbox. In the same way, to perform system fault diagnosis for a WTGS, it only needs to pay attention to parameters such as rotation frequency of the low-speed shaft and resonant frequency of the tower tube of WTGS. According to different components of the WTGS, different feature 
frequencies are selected, and the fault feature frequency triggered by the actual vibration signal is determined by the frequencyselection network, and then, automatic identification of the fault feature frequency of the WTGS is completed as shown in Table 2:

Table 2. Sample table of fault diagnosis
\begin{tabular}{|c|c|c|c|c|c|c|}
\hline $1 \mathrm{X}$ & $2 \mathrm{X}$ & $3 \mathrm{X}$ & $4 \mathrm{X}$ & $5 \mathrm{X}$ & $\ldots$ & Diagnostic result \\
\hline 1 & 1 & 1 & 1 & 1 & $\ldots$ & Loose fault \\
\hline 1 & 1 & $/$ & $/$ & $/$ & $\ldots$ & Misalignment \\
\hline$\ldots$ & $\ldots$ & $\ldots$ & $\ldots$ & $\ldots$ & $\ldots$ & $\ldots$ \\
\hline
\end{tabular}

The type of WTGS components and the code of fault feature are combined together to be encoded and expressed in one word as shown in Figure 5 below:

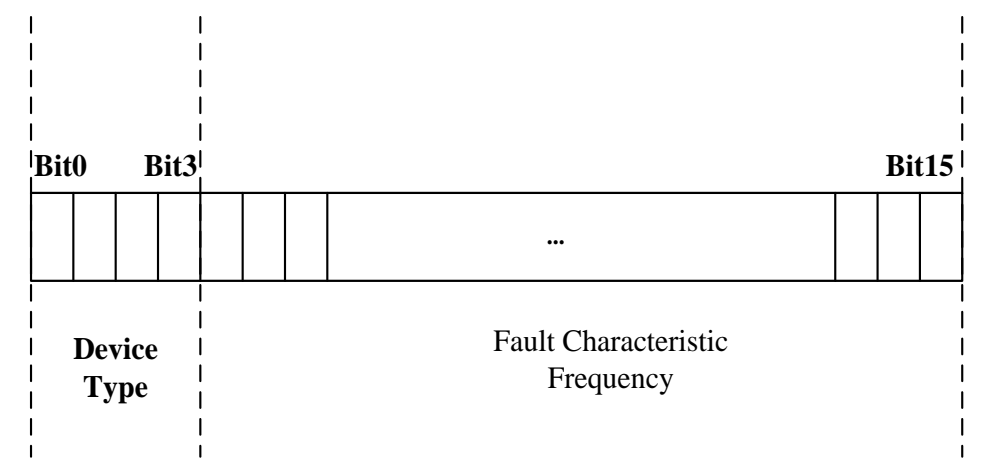

Figure 5. Coding rule of WTGS fault features

As shown in Fig. 5, one word is represented by a total of 16 bits from Bit0 to Bit15, where the bits from Bit0 to Bit3 are used to indicate the type of components of WTGS. For example, the code "0011" represents the generator fore bearing, and "0100" represents the generator rear bearing, as shown in Table 3:

Table 3. Coding table of components
\begin{tabular}{|c|c|c|c|c|}
\hline Component type & Bit0 & Bit1 & Bit2 & Bit3 \\
\hline Nacelle & 0 & 0 & 0 & 1 \\
\hline Tower & 0 & 0 & 1 & 0 \\
\hline Generator fore bearing & 0 & 0 & 1 & 1 \\
\hline Generator rear bearing & 0 & 1 & 0 & 0 \\
\hline Main bearing & 0 & 1 & 0 & 1 \\
\hline Low speed end of gearbox & 0 & 1 & 1 & 0 \\
\hline Coupling & 0 & 1 & 1 & 1 \\
\hline$\ldots$ & $\ldots$ & $\ldots$ & $\ldots$ & $\ldots$ \\
\hline
\end{tabular}

The bits from Bit4 to Bit15 represent different combinations of fault feature frequencies corresponding to the notch filter triggered by the frequency-selection network. For example, the 12-bit code "111111000000" represents that all notch filters corresponding to 1 - to -5 times rotation frequency are triggered, indicating that the fault feature frequency is 1 - to -5 times rotation frequency, and the 12-bit code "110000000000" means that all notch filters corresponding to 1 - and -2 times rotation frequency are triggered, indicating that the fault feature frequency is 1 -and -2 times rotation frequency. Based on the coding rule of component type, the 16-bit code "0100111111000000" indicates that the fault feature frequency of generator rear bearing is 1 - to -5 times rotation frequency, and the 16-bit code "0111110000000000" indicates that the coupling fault feature frequency is 1- and -2 times rotation frequency. After encoding the fault type of the WTGS, the neural network classifier and fault diagnosis expert database can be used to realize automatic fault diagnosis of the WTGS.

\subsection{Neural Network Classifier}

Single-layer neural networks can be used to implement linear classifiers, and multilayers neural networks can be used to implement nonlinear classifiers [10,5,8]. In this paper, a 3-layer neural network is applied to implement nonlinear classifiers, as shown in Figure 6 below, for automatic fault diagnosis of WTGS. 


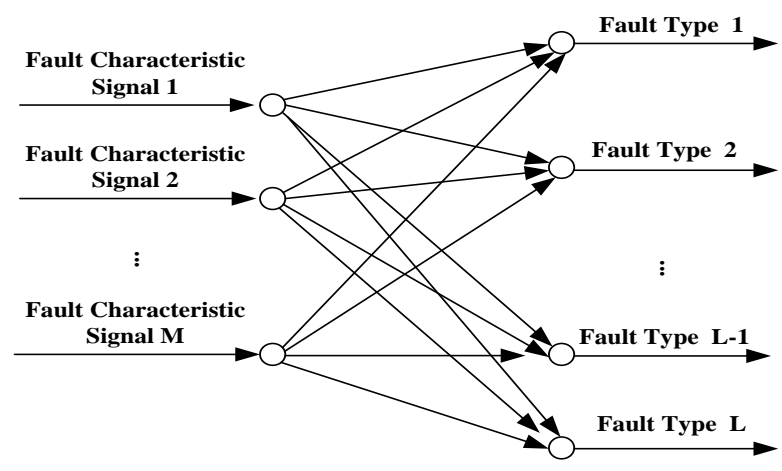

Figure 6. Neural network classifier

The input of the neural network classifier is a 16-bit fault feature code. For example, "0100111111000000" indicates that the fault feature frequency of the generator is 1 - to -5 times rotation frequency; "0111110000000000" indicates that the fault feature frequency of the coupling is 1 - and -2 times rotation frequency.

The output of the neural network classifier is an 8-bit fault type code. The definition rules are shown in Figure 7.

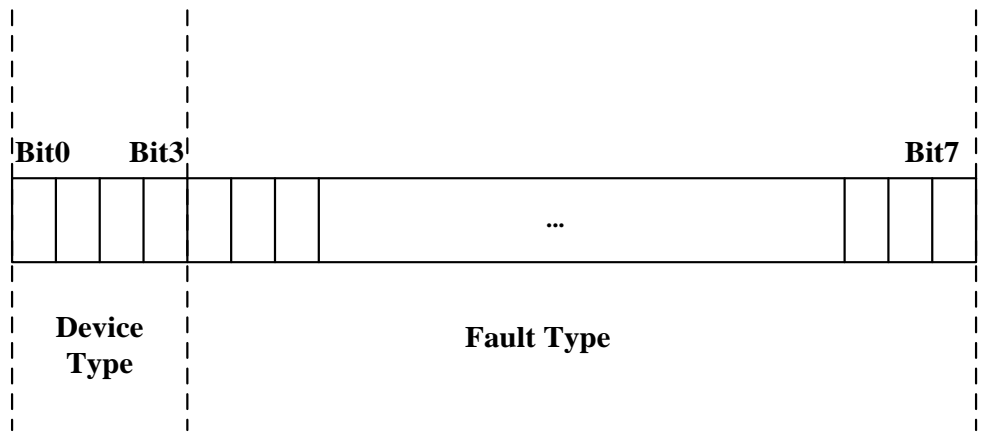

Figure 7. Encoding rule of fault types

\begin{tabular}{|c|c|c|c|c|}
\hline Fault type & Bit4 & Bit5 & Bit6 & Bit7 \\
\hline Generator rear bearing is normal & 1 & 1 & 1 & 1 \\
\hline Generator rear bearing is loose & 1 & 1 & 1 & 0 \\
\hline Generator rear bearing cage fault & 1 & 1 & 0 & 1 \\
\hline Generator rear bearing outer ring fault & 1 & 1 & 0 & 0 \\
\hline Generator rear bearing inner ring fault & 1 & 0 & 1 & 1 \\
\hline Generator rear bearing rolling element fault & 1 & 0 & 1 & 0 \\
\hline Generator stator eccentricity & 1 & 0 & 0 & 1 \\
\hline$\ldots$ & $\ldots$ & $\ldots$ & $\ldots$ & $\ldots$ \\
\hline
\end{tabular}

The fault type shown in Figure 7 is represented by one byte, where the bits from Bit0 to Bit 3 are used to represent the type of components of the WTGS, as shown in Table 3. There are a total of 4 bits from Bit 4 to Bit 7, which can represent 16 fault types of a device. Table 7 shows the generator fault type codes. The output of the neural network classifier is an 8-bit fault type code. For example, "01001110" indicates that the generator rear bearing is loose.

In the neural network, the BP algorithm is adopted to implement one-to-one correspondence between the input of different fault features and the output of different fault types, so as to achieve the automation of fault diagnosis of WTGS.

\subsection{Vibration Intensity Calculation}

The automatic fault diagnosis method for WTGS based on notch filter cannot only achieve an automatic diagnosis of WTGS faults, but also evaluate the degree of failure, as shown in Figure 8. 


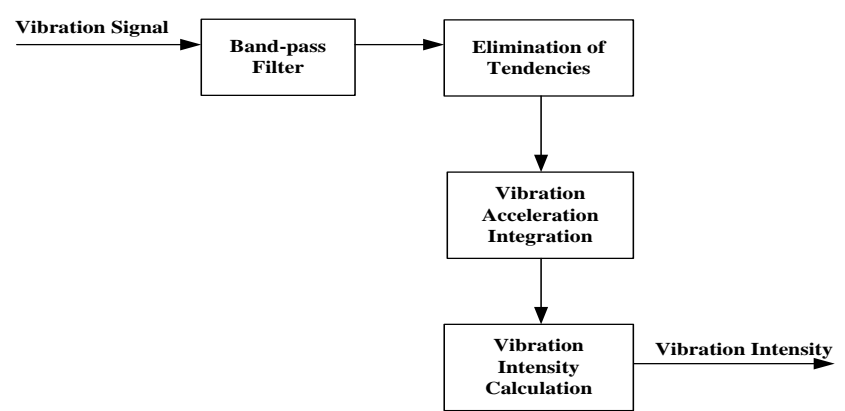

Figure 8. Vibration intensity calculation

Vibration intensity is the physical quantity that describes the vibration intensity of WTGS components and the effective value of vibration velocity. It is also an important parameter to evaluate the vibration of WTGS in fan vibration standard VDI3834. The formula is as follows:

$$
V_{r m s}=\sqrt{\frac{1}{T} \int_{0}^{T} v^{2}(t) d_{t}}
$$

Where $V_{r m s}$ indicates the value of vibration intensity; $T$ represents calculation period; and $v(t)$ represents vibration velocity. When vibration intensity is calculated, the signal frequency range of different components is different. The vibration acceleration signal needs to pass the bandpass filter, and the frequency range of filter is shown in Table 5.

Table 5. Frequency range of vibration intensity calculation of wind turbine componen
\begin{tabular}{|c|c|c|}
\hline No. & Component type & Frequency range \\
\hline 1 & Main shaft of WTGS & $10 \mathrm{~Hz}$ to $100 \mathrm{~Hz}$ \\
\hline 2 & Gearbox & $10 \mathrm{~Hz}$ to $100 \mathrm{~Hz}$ \\
\hline 3 & Generator & $10 \mathrm{~Hz}$ to $100 \mathrm{~Hz}$ \\
\hline 4 & Engine room and tower & $\leq 0.1 \mathrm{~Hz}$ to $10 \mathrm{~Hz}$ \\
\hline
\end{tabular}

The vibration acceleration data collected from the wind power field contains a trend item, which must be eliminated from the vibration acceleration signal. Otherwise, a large error will occur in the calculation of the vibration intensity. The polynomial fitting method is adopted to eliminate the trending item, and the following polynomial is used to fit the trending item in the vibration acceleration signal:

$$
y(k)=a t^{2}+b t+c
$$

Where $t$ is the input parameter; $y(k)$ is the trending item in the vibration acceleration; and $a, b, c$ are the constant terms. The calculation process from the vibration acceleration signal to the vibration velocity signal is shown in Figure 9 below:

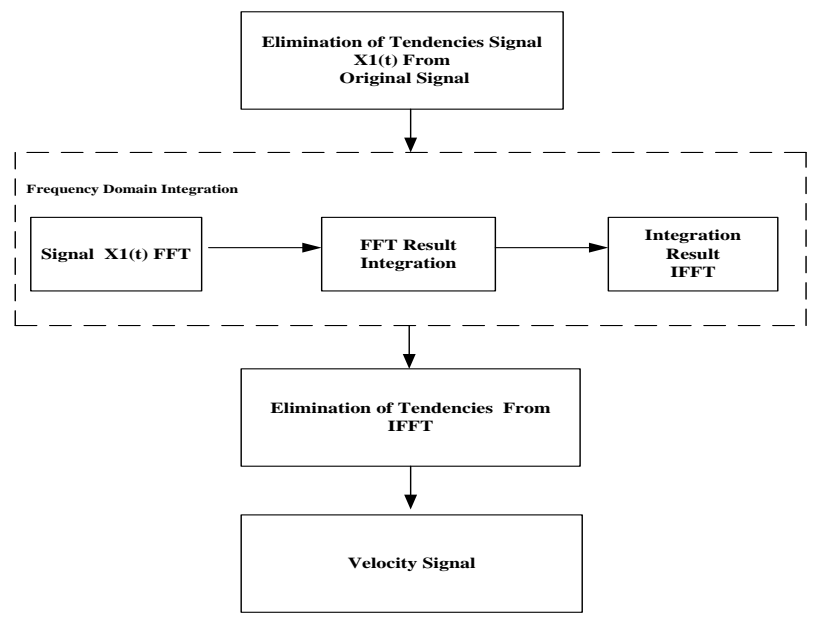

Figure 9. Vibration velocity calculation 


\section{Simulation and Application Case}

\subsection{Field Fault Description}

In a wind farm of Ulanqab City, Inner Mongolia Autonomous Region, the generator rear end of No. 46 WTGS was excessively vibrated under the working condition of $1500 \mathrm{Rpm}$, causing the system to alarm and stop. A vibration acceleration sensor is installed on the generator rear end of the 46\# WTGS to perform data acquisition on the generator running status. The collection parameters are shown in Table 6 below, and the collected data are input to the WTGS's automatic fault diagnosis program to perform a health check on the operating status of generator rear bearing.

Table 6. Data collection parameters

\begin{tabular}{|c|c|c|}
\hline No. & Item & Content \\
\hline 1 & System type & 15MW double-fed WTGS \\
\hline 2 & Sensor type & Vibration acceleration sensor $(0.5 \mathrm{~Hz}-10 \mathrm{KHz})$ \\
\hline 3 & Sensor installation position & Generator rear-end bearing \\
\hline 4 & Sampling frequency & $10 \mathrm{~K} / \mathrm{S}$ \\
\hline 5 & System operating condition & $1490 \mathrm{Rpm}$ \\
\hline
\end{tabular}

\subsection{Design and Simulation of Frequency-selection Network based on Notch Filter}

Generator rear end fault is generally related to generator rotation frequency and frequency doubling, grid frequency, generator bearing inner ring, outer ring, rolling element and cage. Therefore, the parameters of fault feature frequency are as shown in Table 7:

Table 7. Fault feature frequency of the generator

\begin{tabular}{|c|c|c|}
\hline No. & Fault feature frequency & Parameters \\
\hline 1 & Generator rotation frequency (fundamental frequency) & $24.83 \mathrm{~Hz}$ \\
\hline 2 & Power grid frequency & $50.00 \mathrm{~Hz}$ \\
\hline 3 & Fault frequency of inner ring of generator rear bearing & $134.65 \mathrm{~Hz}$ \\
\hline 4 & Fault frequency of outer ring of generator rear bearing & $89.27 \mathrm{~Hz}$ \\
\hline 5 & Fault frequency of the rolling element of generator rear bearing & $58.87 \mathrm{~Hz}$ \\
\hline 6 & Fault frequency of the cage of generator rear bearing & $9.93 \mathrm{~Hz}$ \\
\hline
\end{tabular}

According to the fault feature frequency shown in Table 7, 11 notch filters are designed: the notch frequency is $1-$ to -5 times rotation frequency of the generator, the grid frequency, 2 times grid frequency, and the fault feature frequency of the outer ring of generator rear bearing, fault feature frequency of the inner ring of generator rear bearing, the fault feature frequency of the rolling element of generator rear bearing, and the fault feature frequency of the cage of generator rear bearing, which are shown in Table 8.

Table 8. Type and frequency of notch filter

\begin{tabular}{|c|c|c|}
\hline No. & Notch frequency & Parameter \\
\hline 1 & Rotation frequency and frequency multiplication $(1 \mathrm{X}, 2 \mathrm{X}, 3 \mathrm{X}, 4 \mathrm{X}, 5 \mathrm{X})$ & $24.83 \mathrm{~Hz}, 49.66 \mathrm{~Hz}, 74.49 \mathrm{~Hz}, 99.32 \mathrm{~Hz}, 124.15 \mathrm{~Hz}$ \\
\hline 2 & Power grid frequency and its frequency multiplication(1LF, 2LF) & $50.00 \mathrm{~Hz}, 100 \mathrm{~Hz}$ \\
\hline 3 & Fault frequency of bearing inner ring & $134.65 \mathrm{~Hz}$ \\
\hline 4 & Fault frequency of bearing outer ring & $89.27 \mathrm{~Hz}$ \\
\hline 5 & Fault frequency of bearing rolling element & $58.87 \mathrm{~Hz}$ \\
\hline 6 & Fault frequency of bearing cage & $9.93 \mathrm{~Hz}$ \\
\hline
\end{tabular}

A frequency selection network is designed for the notch frequency shown in Table 8 , and the software framework is shown in Figure 10.

The original vibration signal is shown in Figure 11 below. The upper part is the time domain waveform of the original vibration signal, and the lower part is the waveform diagram of the original vibration signal after FFT. From the figure, it can be seen that the vibration energy is mainly concentrated in the rotation frequency and frequency multiplication of the generator. 


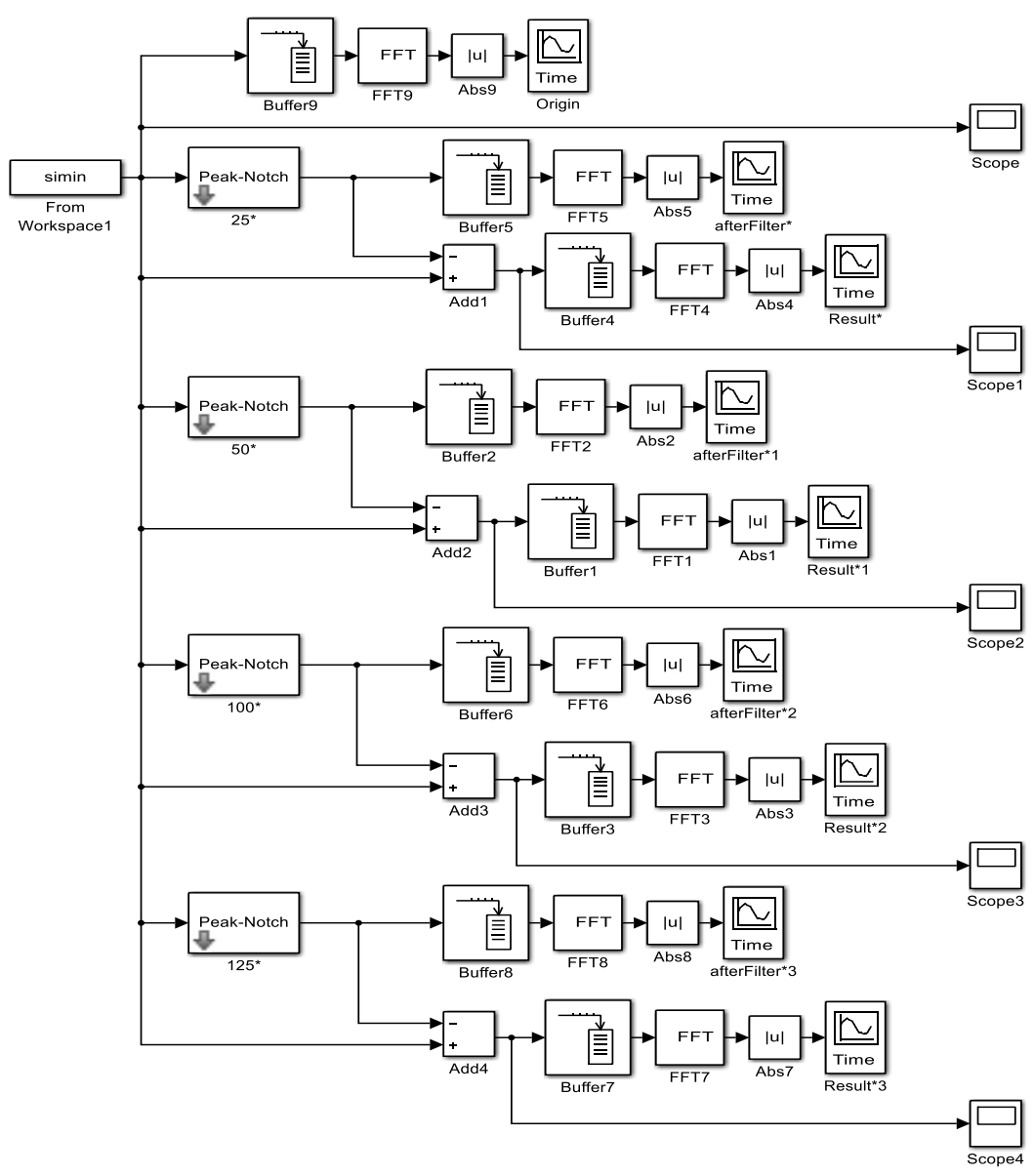

Figure 10. Block diagram of frequency-selection network software based on notch filters
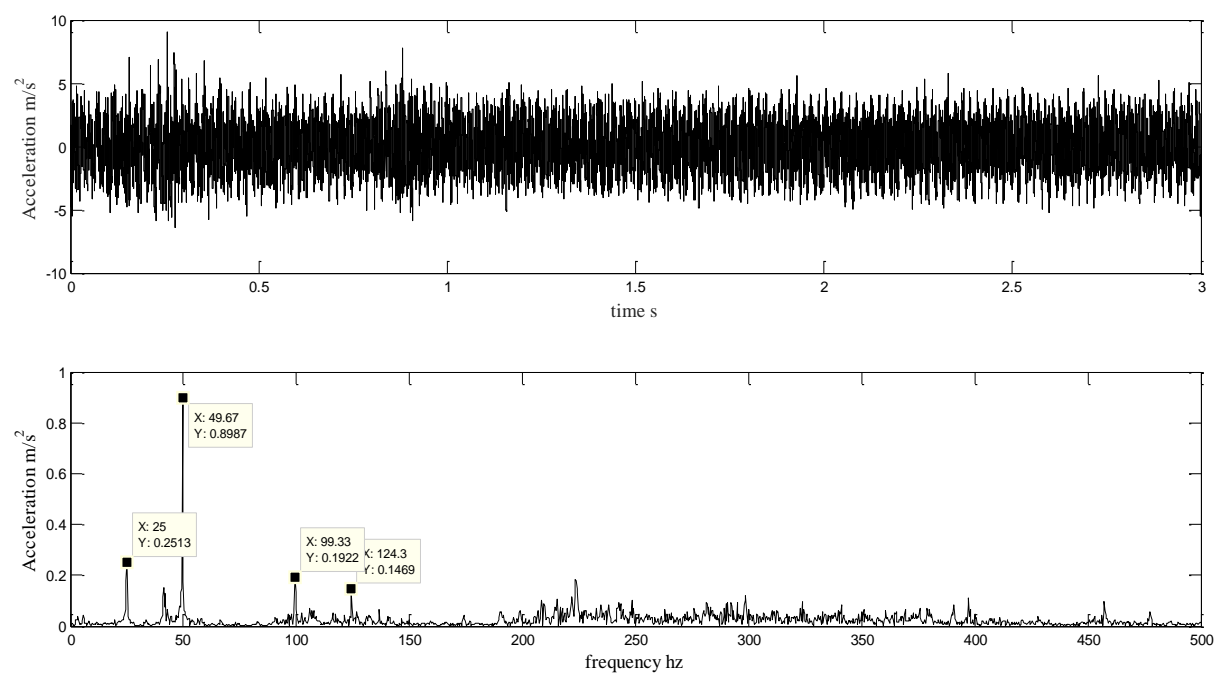

Figure 11. Original vibration signal

The original vibration signal passes through the 11 frequency selection networks shown in Table 8, as well as the frequency-selection network with the notch parameter of the generator rotation frequency and frequency multiplication. The output waveforms are shown in Figure 12 to Figure 15. After the original vibration signal passes through the frequency 
selection network 1, the feature frequency of the 1-time rotation frequency of the generator is extracted. As shown in Figure 12 , the upper part is the time domain waveform of the rotation frequency characteristic signal of the generator system, and the lower part is the frequency domain waveform of the rotation frequency characteristic signal of the generator. Similarly, after the original vibration signal passes through the frequency selection network 1 to the frequency selection network 5, the generator rotation frequency characteristic signal and its frequency multiplication signal are all extracted. The notch frequencies from the frequency selection network 6 to the frequency selection network 11 are the grid frequency, 2 times grid frequency, the fault feature frequency of the outer ring of generator rear bearing, fault feature frequency of the inner ring of generator rear bearing, the fault feature frequency of the rolling element of generator rear bearing, and the fault feature frequency of the cage of generator rear bearing. After the original vibration signal passes from the frequency selection network 6 to the frequency selection network 11, the waveforms are substantially the same, as shown in Figure 16 below.
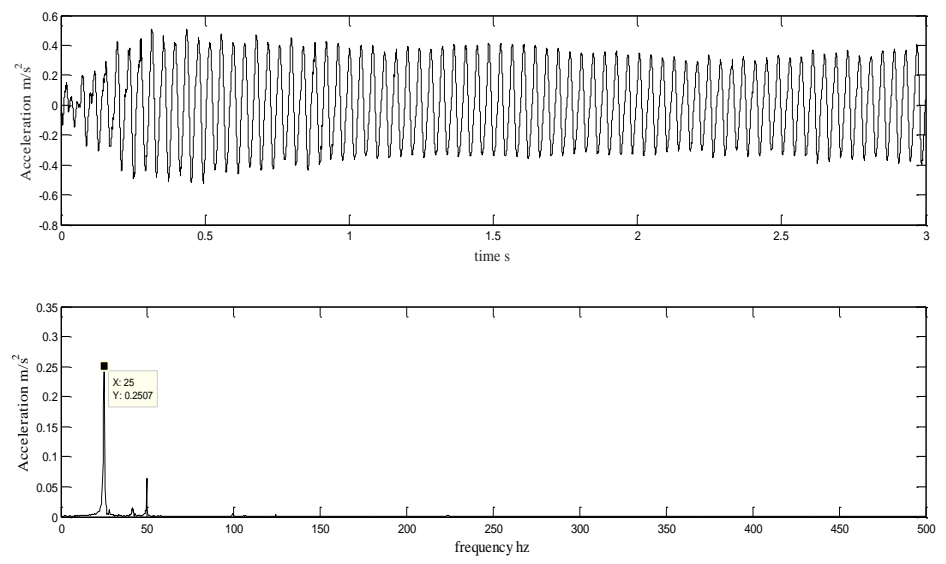

Figure 12. Frequency-selection network 1-1 time rotation frequency
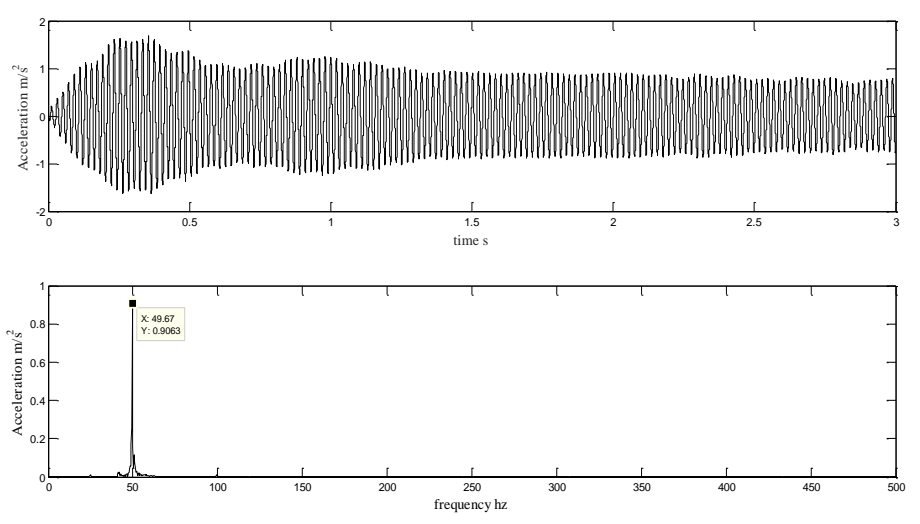

Figure 13. Frequency-selection network 2-2 times rotation frequency
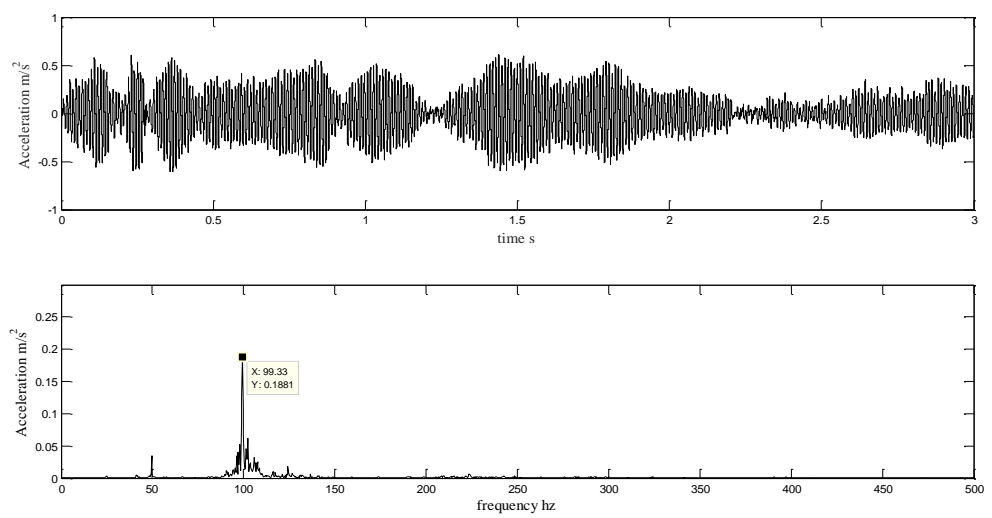

Figure 14. Frequency-selection network 4-4 times rotation frequency 

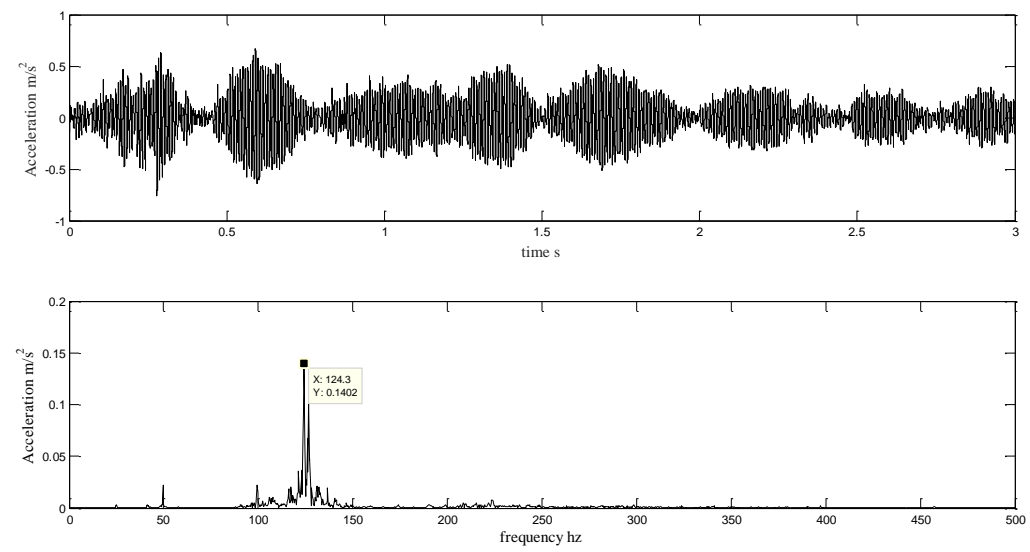

Figure 15. Frequency-selection network 5-5 times rotation frequency
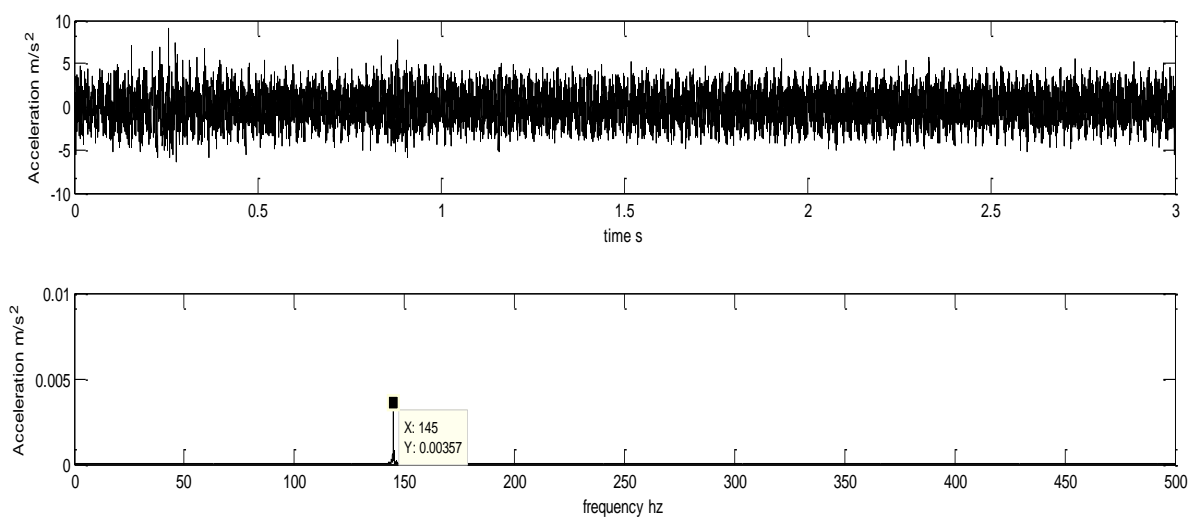

Figure 16. Frequency-selection network 6-11 (fault frequency of power grid and bearing)

Calculate the percentage of the output signals of the frequency-selection network 1 to frequency-selection network 5 to the energy of the original vibration signal. When the percentage is greater than 5\%, it is determined that the frequency-selection network is triggered twice and the triggered frequency-selection network is encoded at the same time. Based on the encoding rule indicated in Fig. 5, the code of the original vibration signal after it passes through the frequency-selection network is: "0100111011000000".

\subsection{Vibration Intensity Calculation}

After the original vibration signal passes through the band-pass filter shown in Table 5 within the frequency range, the trending item is eliminated according to the algorithm shown in Formula (5), and the vibration intensity value is calculated to be 24.67 $\mathrm{mm} / \mathrm{s}$. The vibration intensity threshold specified in the WTGS vibration standard VDI3834 is shown in Table 9.

Table. 9 Alarm threshold table for vibration intensity

\begin{tabular}{|c|c|c|c|}
\hline No. & Component & Warning threshold & Alarm threshold \\
\hline 1 & Engine room and tower & $60.00 \mathrm{~mm} / \mathrm{s}$ & $100.00 \mathrm{~mm} / \mathrm{s}$ \\
\hline 2 & Main bearing & $2.00 \mathrm{~mm} / \mathrm{s}$ & $3.20 \mathrm{~mm} / \mathrm{s}$ \\
\hline 3 & Gearbox & $3.50 \mathrm{~mm} / \mathrm{s}$ & $5.60 \mathrm{~mm} / \mathrm{s}$ \\
\hline 4 & Generator & $6.00 \mathrm{~mm} / \mathrm{s}$ & $10.0 \mathrm{~mm} / \mathrm{s}$ \\
\hline
\end{tabular}

\section{Results}

After the original vibration signal passes through a frequency-selection network and is encoded ("0100111011000000"), when it is input into a neural network classifier, the output of the classifier is "01001110". According to the encoding rule shown in Figure 7, it is determined that the rear bearing of the on-site WTGS has a loosening fault, so the fault degree is serious. 


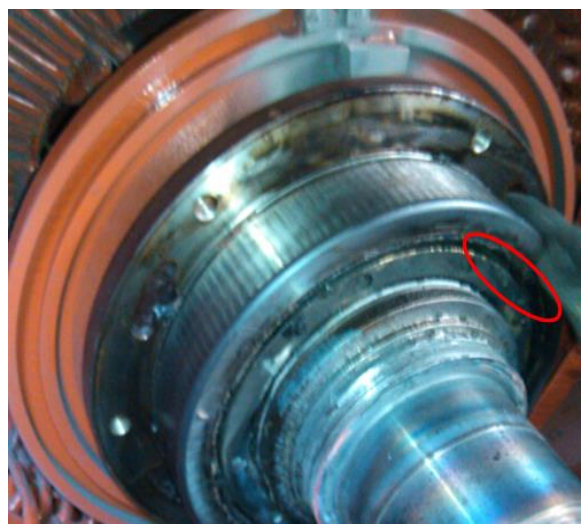

Figure 17. Looseness of generator rear bearing

Based on the conclusions made by the automatic fault diagnosis system, field workers performed an inspection and overhaul of the generator and found that the generator rear bearing did have serious looseness, as shown in Figure 17, proving the feasibility of the method proposed in this paper.

\section{Conclusions}

In this paper, an automatic fault diagnosis method for WTGS based on notch filter is designed, which utilizes the notch filter to weaken specific frequency signals, so as to extract the fault feature frequencies of WTGS components. In this paper, different frequency-selection networks are designed for different components of WTGS, and the frequency-selection network is automatically selected according to the input characteristics of the vibration signal to improve fault diagnosis efficiency. The practical field application shows that the automatic fault diagnosis method proposed in this paper is effective and of important practical significance for realizing batch fault diagnosis of WTGS.

\section{Acknowledgements}

This work was sponsored by the "Eleven Five" National Technology Support Project Funding Scheme (Project No. 2009BAA22B04).

\section{References}

1. Z.Chen, C.Li, and R.V.Sánchez, "Multi-layer Neural Network with Deep Belief Network for Gearbox Fault Diagnosis," Journal of Vibroengineering, vol.17, no.5, pp.2379-2392, 2015

2. P.Chen, L.Song, and H.Wang, "Intelligent Condition Diagnosis Method for Rotating Machinery Based on Probability Density and Discriminant Analyses", IEEE Signal Processing Letters, vol.23, no.8, pp.1111-1115, 2016

3. Center for Intelligent Maintenance Systems, 2017, Retrieved from http://www.imscenter.net

4. S.Devendiran, C.Rajeswari, and B.Sathiyabhama, "Diagnostics of Gear Faults using Ensemble Empirical Mode Decomposition, Hybrid Binary Bat Algorithm and Machine Learning Algorithms," Journal of Vibroengineering, vol.17, no.3, pp.1169-1187, 2015

5. A.Fasana, L.Garibaldi, and A.Tabrizi, "Early Damage Detection of Roller Bearings using Wavelet Packet Decomposition, Ensemble Empirical Mode Decomposition and Support Vector Machine," Meccanica, vol.50, no.3, pp.865-874, 2015

6. S.H.Kim, S.Y.Kim, and I.H.Ra, "Design of Wind Turbine Fault Detection System Based on Performance Curve," in SCIS-ISIS, Kobe, Japan, November 20-24, 2012

7. B.Kim, J.S.LEE, and J.Y.Park, "Design and Application of Condition Monitoring System for Wind Turbines," in Proc. of 11th International Conference on Control, Automation and Systems, Gyeoggido, Korea, Oct.26-29, 2011

8. Y.Kang, C.C.Liao, and C.C.Wang, "Using Bayesian Networks in Gear Fault Diagnosis," Applied Mechanics and Materials, vol.43, no.26, pp.284-287, 2013

9. C.P.Lim, S.Nahavandi, and M.Seera, "Condition Monitoring of Induction Motors: A Review and an Application of an Ensemble of Hybrid Intelligent Models," Expert Systems with Applications, vol.41, no.10, pp.4891-4903, 2014

10. Y.Liu and D.T. Yang, "Bearing Fault Diagnosis Based on Deep Belief Network and Multisensor Information Fusion," Shock and Vibration, pp.1-9, 2016 